

\section{INDICE}

EDITORIAL PERSONAL ÁMBITOS

Apresentação do monográfico. Abordagem qualitativa: olhares e práticas transdisciplinares nas ciências antropossociais

Presentation of the monograph. Qualitative approach: transdisciplinary views and practices in anthroposocial sciences

Ronaldo Nunes Linhares, António Pedro Costa

MONOGRAFICOS MONOGRAPHS

Identidades femininas na rede: as crianças falam!

Female identities on line: children can speak

Marta Maria Azevedo Queiroz

$12-31$

Transição de cuidados de enfermagem: ISBAR na promoção da segurança dos

doentes - revisão scoping

Transition of nursing care: ISBAR in promoting patient safety - scoping review

Ana Rita Esteves Figueiredo, Teresa Maria Ferreira dos Santos Potra, Pedro Ricardo Martins Bernardes Lucas

$32-48$

Integración de elementos cualitativos y cuantitativos en metodología observacional

Integration of qualitative and quantitative elements in observational methodology

M. Teresa Anguera, Angel Blanco-Villaseñor, José Luis Losada, Pedro Sánchez-Algarra

49-70

Atos educativos com oficines de ecografias: uma investigação otobiográfica

Educational acts at echographie's cineliers: an otobiographic research

Silas Borges Monteiro, Anaise Avila Severo

Actuación de las políticas: política como texto y política como discurso

Action of policies: policy as text and policy as discourse

Mónica Rocío Barón

88-104 


\section{ÁMBITOS PERSONALES PERSONAL ÁMBITOS}

Un retrato de la cultura local a través del Periodismo cultural. Análisis comparado de Sevilla y Porto Alegre

A portrait of the local culture through cultural Journalism. Comparative analysis of Seville and Porto Alegre

\section{ARTÍCULOS ARTICLES}

Microsociología del profesor universitario

Microsociology of an university professor

Antonio Fernández Vicente

La pobreza y el discurso de los mass media. Un estudio de la prensa local argentina

Poverty and mass media 's discourse. A study of the Argentine local press

María del Rosario Sanchez, Silvia London

La comunicación no verbal en las elecciones andaluzas de 2018. Comparativa de Susana Díaz y Teresa Rodríguez en el debate de RTVE

Non-verbal communication in the Andalusian municipal elections of 2018. Comparison of Susana Díaz and Teresa Rodríguez in the electoral RTVE debate

María Hernández Herrarte, Patricia Zamora-Martínez

El infoentretenimiento en la televisión de pago, Movistar+ y el canal \#0.

El uso transmedia de sus contenidos de humor

Infotainment on pay television, Movistar+ and channel \# 0 . The transmedia use of its humorous content

Metodología y formación docente cuestiones claves para la integración de las TIC en la educación

Methodology and teacher training as a key issue for ICTs integration in Education 


\section{RESEÑAS REVIEWS}

Aquelarre. Mujeres en la cultura de masas

Coven. Women in mass culture

Regla Ismaray Cabreja Piedra

216-220

Transición ecosocial y principios éticos en el periodismo: una guía para la comunicación de nuevas narrativas

The Eco-social transition and ethical principles in journalism: a guide for the communication of new narratives

Amanda Salazar Torres

Narrativas ecofeministas y mapa de transición ecosocial para medios de comunicación Eco-feminist narratives and ecosocial transition map for the media 


\title{
Actuación de las políticas: política como texto y política como discurso
}

\author{
Action of policies: policy as text and policy as discourse
}

\author{
Mónica Rocío Barón Montaño, Fundación Universitaria San Alfonso. \\ Carrera 25 № 37 - 39, C.P. 111311, Bogotá, Colombia. \\ monica.baron@usanalfonso.edu.co | Orcid: https://orcid.org/0000-0001-7026-4464
}

DOI: https://dx.doi.org/10.12795/Ambitos.2020.i49.06

\begin{abstract}
Resumen
Este artículo resulta de un análisis crítico que fue realizado considerando las discusiones, resultados y conclusiones obtenidas en una investigación desarrollada a partir de un estudio de caso sobre la relación entre política y práctica en el ámbito escolar. Dicha investigación es el resultado del estudio titulado "Método de proyectos: apropiaciones y cuestiones en la contemporaneidad", desarrollado en el Grupo de Estudios sobre Diferencia, Desigualdad y Educación Escolar de la Juventud - DDEEJ, vinculado al Programa de Pos-graduación en Educación de la Universidad del Estado de Rio de Janeiro. La investigación mostró que los estudios de caso en el contexto del enfoque cualitativo pueden aplicarse a la investigación educativa para describir y analizar una unidad social, considerando sus múltiples dimensiones y su dinámica natural, puesto que el trabajo fue desarrollado en una institución escolar perteneciente a la red pública de la ciudad de Bogotá, Colombia. El estudio, que desde un abordaje cualitativo analizó las relaciones entre políticas educativas, instituciones y actores escolares, define la política como "texto" y como "discurso" a partir de las teorizaciones del británico Stephen Ball y colaboradores. Pudo identificarse, en esa problematización, que los textos y los discursos de la política son comprendidos más allá de la suposición de transparencia del lenguaje, en la medida en que constituyen procesos hechos de interpretaciones y traducciones, cuyo sentido es siempre marcado por el tiempo y el espacio en el que ocurren.
\end{abstract}




\begin{abstract}
:
This article results from a critical analysis that was conducted considering the discussions, results and conclusions obtained in an investigation developed from a case study on the relationship between policy and practice in the school environment. This research is the result of the study entitled "Projects Method 'Appropriations and issues in the contemporary world'", developed in the Group of Studies on Difference, Inequality and School Education of Youth $D D E E J$, linked to the Postgraduate Program in Education of the State University of Rio de Janeiro. The research showed that the case studies in the context of the qualitative approach can be applied to educational research to describe and analyse a social unit, considering its multiple dimensions and its natural dynamics, since the work was developed in a school institution belonging to the public network of the city of Bogotá, Colombia. The study, which from a qualitative approach analysed the relationships between educational policies, institutions and school actors, defines the policy as "text" and as "discourse" based on the theorizations of the British Stephen Ball and collaborators. It could be identified, in that problematization, that the texts and discourses of politics are understood beyond the assumption of transparency of language, insofar as they constitute processes made of interpretations and translations, whose meaning is always marked by time and the space in which they occur.
\end{abstract}

Palabras clave: Educación escolar, política educativa, ciclo de políticas públicas

Keywords: School education, educational policy, public policy cycle

\title{
1. INTRODUCCIÓN
}

El siguiente artículo es resultado de un estudio de caso realizado en una escuela situada en la ciudad de Bogotá - Colombia, fruto del trabajo de investigación titulado "Método de Proyectos: apropiaciones y cuestiones en la contemporaneidad". Pretender entender y problematizar académicamente la experiencia de una escuela como escenario de actuación de políticas públicas, en este caso, de la integración de la metodología del trabajo por proyectos, abre discusiones sobre las complejas formas que llevan a las escuelas a enfrentar las múltiples cuestiones suscitadas por las políticas, así como las preocupaciones que su rgen en su propio contexto. La vivencia académica y profesional de la investigadora permitió formular una primera hipótesis a partir de la siguiente cuestión central: la política en las escuelas es un proceso tan diverso y sujeto a diferentes interpretaciones conforme es complejamente configurado, contextualmente mediado e institucionalmente prestado. Con un abordaje cualitativo, la investigación se configuro a partir de un estudio bibliográfico, análisis documental y la utilización de la técnica de la entrevista semiestructurada, ésta realizada con los actores participantes en el estudio y que pertenecen a la Institución Educativa Distrital 
(IED) Pablo Neruda, ubicada en la ciudad de Bogotá. Dentro de esta perspectiva, la investigación apostó que la metodología del trabajo por proyectos es actualmente propuesta como política pública en la educación escolar colombiana y configurada como "texto" y "discurso" en un proceso complejo que registró múltiples traducciones. El abordaje cualitativo de la investigación se configuró en diálogo con la teorización del ciclo de políticas formulada por el británico Stephen Ball y colaboradores, abordaje que no solamente delineo el desenvolvimiento, tanto del estudio bibliográfico como de la investigación empírica que configuró el estudio, como también la propia cuestión central de la investigación y su respuesta hipotética.

\section{PLANTEAMIENTO DE LA INVESTIGACIÓN: OBJETIVOS, HIPÓTESIS Y METODOLOGÍA}

El enfoque metodológico que se consideró más apropiado en la presente investigación empírica fue el estudio de caso, que se justificó por proporcionar una visión profunda de los procesos educativos al centrarse en un contexto específico en el que se llevan a cabo dichos procesos (Stake, 2007). En ese sentido, el estudio de caso se configuró como una modalidad de investigación pertinente, que, en comparación con las otras modalidades de investigación, tiene la ventaja de, como señalan Laville y Dionne (1999, p. 156), ofrecer la posibilidad de ahondar en un aspecto, porque los recursos se concentran en el caso focalizado, considerando también que, según lo justificado por André (2013), si "el interés es investigar fenómenos educativos en el contexto natural en el que ocurren, los estudios de caso pueden ser instrumentos valiosos [...]"(p. 97).

Por lo tanto, centrar el estudio en una escuela en particular, el Colegio Pablo Neruda, representó una oportunidad para el contacto directo con cada uno de los contextos que configuraron esta investigación, brindando posibilidades para describir acciones y comportamientos, capturar significados, analizar interacciones, así como comprender e interpretar. En síntesis, estos sentidos constituyeron una gama de alternativas de análisis que auxiliaron a problematizar cómo la propuesta de la metodología del trabajo con proyectos (MTP) puede ser reformulada en el contexto educativo colombiano contemporáneo.

Para problematizar algunas de las formas por las cuales la MTP está siendo hoy interpretada en el Colegio Pablo Neruda, la investigación se basó en el enfoque del ciclo de políticas formulado por Stephen Ball y colaboradores como una contribución relevante, estableciendo para ello algunas cuestiones y objetivos que nortearon el estudio.

En este camino fue necesario reconocer que, a lo largo de su trayectoria, el trabajo por proyectos ha atendido diferentes intereses en el campo del trabajo pedagógico, consolidando experiencias que se han configurado en la materialización de una dinámica que ha venido evolucionando y alcanzando diferentes enfoques, evidenciando aspectos únicos de cada experiencia vivida pero complementarios entre sí, incluso si se sitúan en instituciones o tiempos distantes. 
Esta trayectoria puede identificarse a partir de opiniones de autores como Bleeke, 1968; Hernández, 1998a, 1998b; Kliebard, 2011; Knoll 1997; Miñana, 1999; Moore, 1988; cuyos abordajes profundizan el tema del trabajo con proyectos y que, aunque representen pensamientos configurados en función de marcadas diferencias en cuanto al campo del conocimiento en el que se insertan, así como en el espacio y el tiempo en el que son abordados, en algunos aspectos aún mantienen relación.

Podríamos comenzar refiriéndonos a la conceptualización del término en sí mismo, señalándolo como una propuesta que, según el enfoque de Dewey y Kelpatrick (Knoll, 2012), tendría el objetivo de ultrapasar los límites del aula e invitar al alumno a reflexionar sobre los problemas de su día a día. Sin embargo, como lo señaló Miñana (2008, p. 2), el discurso de la literatura educativa y los órganos institucionales de educación, en lo que tiene que ver con el trabajo con proyectos en Colombia ha sido poco abordado en la investigación académica.

En consecuencia, la inclusión del tema de la MTP en el campo de la política pública educativa despierta el interés de esta investigación, que busca contribuir a la construcción de una respuesta académica a la siguiente pregunta: ¿cómo y por qué está siendo resignificada la propuesta de la MTP como estrategia en el contexto educativo colombiano actual?

En este movimiento, nos arriesgamos a la hipótesis de que la política en las escuelas es un proceso tan diverso y sujeto a diferentes interpretaciones conforme es complejamente configurado, contextualmente mediado e institucionalmente prestado. Por lo tanto, la metodología del trabajo por proyectos se propone actualmente como política pública en la educación colombiana en un proceso complejo que registra disputas por traducciones más cercanas a perspectivas críticas, liberales - escolanovistas y neoliberales.

No se puede desconocer que la aproximación a la política exige, entre otros aspectos, avanzar en el análisis de los discursos que circulan y dan sentido a su problematización y que, en el caso aquí abordado, son entendidos más allá de la suposición de la transparencia del lenguaje, en la medida en que significan un proceso hecho de interpretaciones y de traducciones. Desde esta perspectiva, las interacciones dialógicas, opiniones, discursos y juicios configurados a partir de las interacciones con los actores que participan en los múltiples espacios de concretización de la MTP, las formas de significar sus experiencias, de establecer relaciones, así como la forma de involucrarse en la participación de la propuesta pedagógica, son cuestiones constitutivas de la política.

A la luz de las cuestiones ya mencionadas, optamos, como se anunció, por el sesgo teórico del abordaje del ciclo de políticas formulado por Stephen Ball y colaboradores (Bowe, Ball y Gold, 1992; Ball, 1994). Cabe destacar que la reflexión sobre tal abordaje es relevante en la coyuntura colombiana, ya que, según Rincón (2010, p. 36), el campo de investigación en políticas educativas en Colombia aún no se ha consolidado en términos de referentes analíticos consistentes, debido a la "[...] escasa información sobre las políticas educativas actuales en Colombia, en términos de análisis y comprensión de sus efectos prácticos en la 
transformación del sistema educativo". A partir del presupuesto de que las políticas, entendidas en su complejidad y caracterizadas por elementos inestables y contradictoriosestán en permanente movimiento a través del tiempo y el espacio, el abordaje del ciclo de políticas nos ayudó a comprender los objetivos la investigación se trazó.

\section{CAMPO EMPÍRICO}

Partiendo de la premisa de que las políticas son interpretadas y traducidas por diversos actores dentro del ambiente escolar, en lugar de simplemente implementadas, pero también considerándolas como procesos discursivos que son complejamente configurados, contextualmente mediados e institucionalmente prestados (Ball, Maguire y Braun, 2016), buscamos aprovechar en este estudio las estrategias usualmente utilizadas en estudios con paradigmas interpretativos.

En este sentido y buscando dar cuenta de los objetivos señalados para esta investigación, fue necesario considerar la contribución de diversas herramientas para la producción y el análisis del material empírico de la investigación, sea a través de entrevistas semiestructuradas con los actores participantes del Colegio Pablo Neruda, mediante una mirada atenta a la revisión bibliográfica y al análisis de los textos y documentos que abarcan el abordaje de la MTP o mediante el análisis de las discusiones desarrolladas en las reuniones de maestros y gestores con respecto a dicha propuesta. Después de estas consideraciones, se presentan los procedimientos de investigación que contribuyeron a la consolidación del estudio.

\subsection{Entrevista: bases teóricas}

Al hacer una aproximación a lo que se entiende como entrevista, presentamos la definición de Arfuch (1995, p. 25), según la cual, la entrevista puede ser clasificada como un género discursivo que, desde una perspectiva multidisciplinaria, abordará en primer lugar la situación comunicativa, regida por el intercambio dialógico, sus participantes, su proximidad con la conversación cotidiana, los usos del lenguaje, sus infracciones, lo predecible e impredecible que tiene este juego intersubjetivo de verdad. En segundo lugar, la autora llama la atención sobre la forma en que interviene la afectividad, la expresión de los sentimientos, los personajes que se dibujan en esta escena (entrevistadores y entrevistados). Y finalmente, se refiere a las "historias" que se narran en la evolución del diálogo, que nos permiten acercarnos a la literatura y, sobre todo, a los géneros biográficos.

Es, pues, en este movimiento que Arfuch (2010) retoma la expresión del "espacio biográfico" de Philippe Lejeune, para profundizar en la reflexión sobre la construcción de una esfera de interacción particular, que se pone en movimiento en las dinámicas de conversación que caracterizan las entrevistas, las historias de vida, autobiografías y, en general, cualquiera de los métodos que subyacen en el trabajo para recuperar el testimonio del otro. En otras palabras, el acceso a la experiencia de los participantes de la entrevista posibilitó la reflexión 
en torno a las especificidades del mundo social en el que están inmersos y su relación con la resignificación de la MTP. O lo que es lo mismo, el privilegio de conocer la experiencia de los participantes con la estrategia pedagógica que nos convoca, abriendo posibilidades para una mejor comprensión de la escuela y sus significados en los tiempos contemporáneos.

En este escenario, los entrevistados, según Arfuch (2010, p. 241) son reconocidos como "hombres y mujeres comunes [que hacen parte del cotidiano del colegio], [y que] se convierten en personajes, [...] y se transforman en testimonios o actores" y la entrevista como medio para, en palabras de la autora, dar voz a los protagonistas. Sin embargo, es importante señalar que, como se mencionó en líneas anteriores, la consideración de los entrevistados en este estudio no es simplemente la de informantes, incluso si sus contribuciones son fundamentales para lograr los objetivos propuestos en esta investigación, sino como interlocutores, como actores cuyas experiencias y narrativas pueden contribuir a nuestro análisis, constituyentes de un universo de voces externalizadas en el campo del estudio en foco.

Por lo tanto, en la búsqueda de un mejor conocimiento sobre los significados atribuidos por los actores escolares que pertenecen al Colegio Pablo Neruda con respecto a la experiencia con las políticas educativas, particularmente la MTP, la investigación integró algunas cuestiones que se plantearon a los participantes.

Es necesario, en este punto, presentar el perfil de los actores involucrados en la investigación, a saber: el director de la escuela, dos maestros líderes del proyecto, ocho maestros, diez estudiantes del segundo año de educación secundaria, diez estudiantes del tercer año de educación media y ocho estudiantes egresados.

El contacto con estos autores se realizó a través de entrevistas semiestructuradas, a partir de un guion flexible, pero buscando cubrir los puntos considerados esenciales en una "necesaria interacción entre los presupuestos teórico-metodológicos a ser validados y los resultados esperados, de acuerdo con un marco de conocimiento previo, [considerando que] el corpus construido debe ser, incluso según los parámetros cualitativos, representativo" (Arfuch, 2010, p. 249, énfasis agregado). El guion de la entrevista condujo a una serie de preguntas previas, pero en la conversación otras cuestiones fueron planteadas de acuerdo con el diálogo y las respuestas expresadas, evocando la entrevista como un espacio biográfico abierto al diálogo donde ocurren numerosas transformaciones, con mayor o menor repercusión en los procesos de significación.

Fueron presentadas a los entrevistados, preguntas sobre su visión general de lo que significa la MTP; sobre la caracterización de la MTP en el escenario académico del Colegio; sobre la posibilidad de reconocer, dentro de las experiencias ocurridas en varios momentos, los fundamentos de esta estrategia pedagógica; sobre la participación de alumnos y docentes en esta experiencia. También se hicieron preguntas sobre la mediación entre la propuesta de la MTP y la relación profesor-alumno, así como la relación de los estudiantes con el 
conocimiento, con el fin de identificar la caracterización de esa estrategia pedagógica en la dinámica escolar del Colegio. Se formularon preguntas sobre lo que les gustó y no les gustó frente a la dinámica desarrollada y los resultados que se estaban alcanzando o se lograron durante el transcurso de la experiencia.

El análisis de la información obtenida a través de las entrevistas se realizó por medio del método de análisis del discurso, tal como está delineado en las teorizaciones de Ball y colaboradores (2016) siguiendo a Foucault, como una posibilidad que, mediante la incorporación de significados y el uso de proposiciones y palabras, construyen ciertas posibilidades de pensamiento. En palabras del autor, "Los discursos pueden parecer un pequeño folleto", dice Foucault (1999, p. 11), pero las prohibiciones a las que él está sujeto pronto revelan sus vínculos con el deseo y el poder " (Ball, 1994, p. 22).

\subsection{Análisis del discurso}

Con el fin de centrarnos en un análisis detallado y profundo del material empírico, abordamos la contribución del análisis del discurso (AD) no reducido a disciplina metodológica, técnica o ciencia, sino como un ejercicio de pensamiento que busca aclarar las singularidades lingüísticas del discurso como producción social teniendo en cuenta que, como lo refiere Maingueneau (2015), el análisis del discurso prácticamente puede designar cualquier cosa (toda la producción del lenguaje puede considerarse 'discurso'), dando la posibilidad de observar los acontecimientos del cotidiano como manifestaciones semióticas, es decir, materializadas en la actividad del lenguaje y dotadas de significados.

De hecho, una de las grandes contribuciones del $A D$ en esta perspectiva fue la de ofrecer apoyos analíticos que nos llevaron a pensar que todo discurso posee significado en el lenguaje cotidiano. Por lo tanto, toda situación en la que encontramos personas conversando, debatiendo, exponiendo ideas, es decir, donde se pronuncian palabras, oralmente o por escrito, o incluso a través de formas no verbales del lenguaje, en todas estas prácticas lingüísticas, hay discurso.

Es necesario seguir, en el ámbito del $A D$, rastros que nos permitan comprender mejor las múltiples singularidades desencadenadas en la actividad del lenguaje. Con este fin, exploraremos de manera breve las contribuciones teóricas y analíticas desde la perspectiva de Foucault, en los discursos, refiriendo el enfoque presentado también por Ball y colaboradores (2016) en la teorización sobre la actuación de las políticas, en donde dicha perspectiva se entiende como "[ ...] el conjunto de condiciones bajo las cuales se ejerce una práctica, según las cuales esa práctica da origen parcial o totalmente a nuevas declaraciones, y según las cuales ella puede modificarse" (Foucault, 1986, pp. 208-209). Este tipo de afirmación adquiere significado e importancia para el AD aquí pretendido, en la medida en que, en estos términos, la MTP puede considerarse como representaciones de conocimiento y poder (conjuntos de textos, eventos, prácticas) que convergen con elementos internos y externos a la dinámica del Colegio Pablo Neruda y cargan significados. 
Situar esta perspectiva en un intento de entender la MTP como una política configurada dentro de un proceso de enfrentamientos, de establecimiento de enunciados, de delimitación de interlocutores, de lucha por sentidos, entre otros, nos llevó a los abordajes de Ball, Maguire, y Braun (2016, p. 173), para quienes las políticas son "[...] conjuntos de textos, eventos y prácticas que hablan con procesos más amplios de la escuela". Desde esta perspectiva, proponiendo un diseño de investigación cualitativa inductiva, cuyo método principal de análisis combina la teoría y el empirismo, los autores buscan comprender, no las políticas en sí, sino el cómo y qué de la actuación de políticas, para llegar a una Teoría de la actuación, utilizando los conceptos de Foucault. En palabras de los autores, "[...] el trabajo de Foucault nos brindó provocaciones para pensar de manera diferente sobre el trabajo de la política" (Ball, Maguire, Braun, 2016, p. 191).

Como se ha señalado, el análisis del discurso en la perspectiva aquí definida tiene algunas repercusiones, por lo que, en el modelo analítico así propuesto para la comprensión de las políticas, como se mencionó en líneas anteriores, se asume la noción de política como texto y discurso.

En base a esta teorización, Ball y sus colaboradores intentan resaltar las diversas posibilidades de análisis que permiten distinguir no solo múltiples lecturas, debido a la pluralidad de lectores, sino también reconocer las influencias, tensiones, intenciones y negociaciones que implican el reconocimiento, o no, de algunas voces (representadas en textos y discursos), en virtud de comprender las innumerables formas en que la política se pone en práctica en un escenario escolar.

En el proceso de acercamiento a las políticas públicas propuestas desde la concepción foucaultiana, de la cual estos autores se apropian, el AD tuvo el potencial de auxiliar a esta investigación en el interés de concebir el lenguaje más allá de un conjunto de signos con significantes asociados con ciertos significados. De modo que, lo que interesó, como lo destacó Foucault (1999 apud Tello, Mainardes, 2012, p. 16), fue comprender que "[...] el discurso no se centra en las palabras que usamos para designar las cosas, sino por el contrario, en la forma en que las palabras construyen las realidades a las que se refieren como un ejercicio de poder ".

Desde esta perspectiva, el AD exige, como se ha dicho, escapar de la interpretación simple de lo que estaría detrás de esos materiales, buscando explorarlos al máximo, ya que representan una producción histórica y política en la que las palabras también son construcciones y, donde el lenguaje se delinea como constitutivo de las prácticas. Según Fischer (2001, p. 198), una de nuestras tareas como investigadores analistas del discurso es, por lo tanto, "tratar de separarnos de un aprendizaje largo y eficaz que todavía nos hace mirar los discursos apenas como un conjunto de signos, como significantes que se refieren a ciertos contenidos, con tal o cual significado [...] ". Destacamos entonces que entender la política como texto y la política como discurso implica procesos complejos, ya que no se limitan a una representación estática, sino que envuelven poder, intereses e historia. Sus potencialidades 
consisten en la amplitud de una producción de sentidos que se incorporan a la política en formación.

La opción por el AD como propone Foucault en la lectura de Ball lleva a concebir el discurso como "el [...] conjunto de regularidades, un rastro de la 'frontera histórica' de un evento [...] o el conjunto de condiciones, de acuerdo con el cual se ejerce una práctica, según el cual esa práctica da lugar a nuevas declaraciones" (Foucault, 1986, p. 208 apud Ball, Maguire y Braun, 2016), aportándonos herramientas para identificar un conjunto de discursos que representan la posibilidad de comprender cómo los diferentes grupos de actores que participan en la investigación interpretan y actúan la MTP, apropiándose, o no, de los procesos de la reforma, así como de las alteraciones y adaptaciones del texto de la política que se hacen efectivas en su aplicación.

En esta investigación, optar por el enfoque de los fundamentos teórico-metodológicos inscritos en el ciclo de políticas de Ball y colaboradores $(2014,2016)$ y en el análisis que se propone a partir de la lectura que el autor hace de Foucault y Fairclough sobre el AD como una práctica social, nos permitió trazar un camino para abordar el corpus de la investigación pertinente con el abordaje de la política pública movilizado en esta investigación.

\section{POLÍTICA EDUCACIONAL, DISTINCIÓN ENTRE “TEXTO” $Y$ “DISCURSO”}

La sociología política de Stephen Ball se caracteriza por centrar su análisis en la conceptualización y comprensión de la política, es decir, tratar de explicar qué son y cómo se pueden investigar sus trayectorias y efectos en el campo educativo. Por lo tanto, esta investigación abordó el enfoque del ciclo de políticas como teoría que propone "[...] 'transformar' la política [educativa] en un proceso, tan diverso y repetidamente contestado y lo sujeto a diferentes 'interpretaciones' en la medida en que es enscenado (puesto en escena, en actuación) de maneras originales y creativas dentro de las instituciones [...]" (Ball, Maguire, Braun, 2016, p. 13).

Desde su perspectiva, el análisis de las políticas educativas permite "dar voz" a los actores desconocidos y hacer visibles las relaciones de poder-conocimiento en escenarios específicos y locales (Ball, Maguire, Braun 2016). En consecuencia, de acuerdo con el enfoque propuesto por el autor, la política puede entenderse simultánea y complementariamente como "texto" y "discurso", ya que una está implícita en la otra. Por lo tanto, sus abordajes ofrecen herramientas conceptuales y teóricas, dinámicas y flexibles que permiten un análisis cualitativo de la trayectoria o ciclo de las políticas educativas, consistente con la comprensión de la política "como textos y 'cosas' (legislación y estrategias nacionales), pero también como procesos discursivos que son configurados de manera compleja, mediados contextualmente y prestados institucionalmente" (Ball, Maguire, Braun, 2016, p. 13). 
En virtud de ese juego de interpretación, comprender las complejas formas en que la propuesta de MTP está siendo resignificada en el escenario contemporáneo es un proceso que, visto como discurso y como texto, implica juntamente referir perspectivas presentes en un campo de ideas que ha permeado su trayectoria, también atravesada por una red compleja de múltiples influencias en el marco de las relaciones sociales y de poder que la circunscriben como una posible práctica en el escenario escolar.

Así, la MTP como discurso se configura como un proceso de enfrentamientos, demarcación de interlocutores, establecimiento de enunciados y lucha de sentidos en su concretización; y comprenderla como texto implicó reconocerla como política dialógica y favorecedora de representaciones, reapropiaciones y recontextualizaciones que son configuradas de maneras complejas en razón a la pluralidad de interpretaciones. Por lo tanto, entendemos que la MTP como propuesta educativa se presenta vinculada a diversos modelos políticos, construidos de ideas, normas y padrones de relación social.

La lectura del corpus empírico construido por la investigación apunta marcas, más o menos hibridadas, de perspectivas teórico-políticas que pensamos poder identificar como liberalescolanovistas, críticas y neoliberales.

Por lo tanto, en una visión global de la MTP en el ámbito escolar, se puede observar que aquellos que se han referido a los orígenes de la MTP colocan a John Dewey como su inspirador y William H. Kilpatrick en la posterior materialización de propuesta.

Dewey, en un abordaje compartido por Kilpatrick, entendió que la educación es un proceso en el cual el ser humano está inserido de acuerdo con sus experiencias. Por lo tanto, su concepción de la escuela implicaba considerar al estudiante como un ser activo. El método de enseñanza que el propuso es indirecto, porque lo que se espera es el descubrimiento reflexivo y competente. De la misma forma, desde su perspectiva, los campos de estudio no se conciben de manera tradicional, ya que el método se centra en los problemas, lo que lo hace incompatible con la división de disciplinas escolares.

En esa medida, para el propio Dewey, el programa escolar "debe tener en cuenta la adaptación de los estudios a las necesidades de la comunidad, de la vida existente; debe hacer una selección para mejorar la vida que vivimos en común para que el futuro sea mejor que el pasado" (Dewey, 1979, p. 206).

En términos políticos, se valora el individuo y la actuación política del estudiante en términos típicamente liberales. Es de destacar que la noción de interés del estudiante, central para las propuestas pedagógicas de Dewey y Kilpatrick, tiene un sesgo más psicológico que sociocultural en esta perspectiva.

Por lo tanto, se denominó como liberal-escolanovista la enunciación de observación, documento o entrevista que se refiere más directamente a tales valores y proposiciones, distinguiéndose del enunciado neoliberal, considerando que, como lo mencionaron Saraiva y 
Veiga-Neto (2009, p. 188), "El liberalismo, desde la década de 1980, ha sido resignificado en forma de neoliberalismo".

Aludimos en este punto al neoliberalismo, ya que en las últimas décadas el entorno políticodoctrinal global se ha polarizado en torno a sus diferentes formas. Sin embargo, de acuerdo con Ball (2014, p. 25), el neoliberalismo "es uno de esos términos que se usa de manera tan amplia y vaga que corre el riesgo de perder su sentido". Además, el autor, citando a Wood (1997 apud Ball, 2014, p.25), todavía se refiere al neoliberalismo como un conjunto contradictorio de prácticas que se organizan como base para "la universalización de las relaciones sociales basadas en el mercado, con la presencia correspondiente en casi todos los aspectos de nuestras vidas, desde el discurso y / o la práctica de la mercantilización, la acumulación de capital y la generación de lucros".

En las políticas públicas para la educación, lo característico del neoliberalismo es proponer una visión economicista vinculada a la primacía del mercado como la visión más apropiada y la única legítima para orientar las decisiones en el campo educativo y considerar la educación como una mercancía. Philip Coombs (1971), reconoce que, en el neoliberalismo la educación en esencia no es más que una mercancía que se comercializa en un mercado específico en el que, con algunos lemas políticos breves, se sostiene una serie de medidas económicas complejas. A partir de esta idea central, los conceptos que provienen de fuentes teóricas y tradiciones muy diferentes se resignifican por el neoliberalismo: democracia, libertad, igualdad, justicia, mérito, educación, Estado, público y privado, autonomía, descentralización, calidad, equidad, responsabilidad, participación, competencia, educación permanente, sociedad educativa. Como afirma Tadeu Da Silva (1997, p. 178), "[...] el discurso de la educación neoliberal depende del establecimiento de un sistema lingüístico que gira en torno a palabras, conceptos, expresiones y relaciones que aparentemente hacen que sea muy difícil expresar una opinión contraria".

Finalmente, es relevante mencionar aquí la perspectiva conocida como crítica, cuya expresión, a pesar de presentar una gran polisemia (Candau, 2003), proviene en gran medida del conocimiento académico de autores como Henry Giroux, Ira Shor, Michel Apple, Paulo Freire, Antonio Gramsci, John Dewey, Michel Foucault, Pierre Bourdieu, entre otros. Estos teóricos participaron en estudios relacionados con temas de poder, dominación, opresión, justicia, igualdad, identidad, conocimiento, cultura entre otros, contribuyendo a la profundización de los problemas educativos que enfrentamos hoy y suscitando un fuerte movimiento que genera ideas y prácticas, orientadas a la formulación de políticas públicas, así como a la renovación de las prácticas pedagógicas en las escuelas.

Es necesario reconocer, dentro de esta corriente, la existencia de diferentes concepciones y principios pedagógicos que se manifiestan moldeándola e intentan guiar la acción pedagógica de los actores educativos, así como reconocer la forma como han configurado diferentes líneas de pensamiento que la enriquecen y en la mayoría de los casos la complementan. Sin embargo, en este estudio, movilizamos tal encuadramiento como referencia a enunciados 
que, de manera amplia, se aproximan de las características enumeradas por Candau, en contraste con aquellas que marcan las otras perspectivas que, en esta investigación, identificamos en disputa por los sentidos que la MTP asume en el escenario educativo colombiano.

\section{RESULTADOS Y CONCLUSIONES SOBRE LA INVESTIGACIÓN}

En síntesis, a partir de los resultados de las diversas fases del estudio, se puede afirmar que, para el análisis de los diversos contextos de políticas, las teorizaciones de Ball y colaboradores (2016, p.12) sobre "cómo las escuelas hacen políticas, específicamente sobre cómo las políticas se tornan vivas y actuantes [o no] en las escuelas", propician una comprensión de las políticas como intervenciones textuales, enfatizando su carácter de incompletitud y complejidad. Según los autores, lo que se concibe por política no es simplemente comprendido como texto, sino también como un proceso discursivo, lo que contribuyó para el desarrollo del análisis de esta investigación, al presentar la noción de que una política, además de procesos y efectos, es texto y también discurso. Y estas dos conceptualizaciones, texto y discurso, están mutuamente implicadas, como se mencionó anteriormente, y fueron retomadas en complemento a lo ya presentado.

Según los análisis de autores colombianos como Jaime Jaramillo, Carlos Miñana, Martha Cecilia Herrera, Marco Raúl Mejía, Julián de Zubiria, quienes, entre otros, nos ayudaron a comprender mejor la adaptación de la MTP a las recientes políticas públicas colombianas, en Colombia se presenta escasa producción académica que dé cuenta de trabajos de sistematización de esta propuesta metodológica, lo que hace aún más difícil problematizar su actuación.

Sin embargo, entre las observaciones, se destaca la referencia hecha por los autores a una tendencia común en las políticas adoptadas por los gobiernos de las últimas décadas y que estaría sujeta a las prescripciones del economicismo, lo que llevaría a observar un proceso continuo y de cambios visibles, que viene afectando a la sociedad colombiana. Por lo tanto, los planes gubernamentales y las políticas públicas estarían buscado más una respuesta a las demandas de agencias internacionales como el Fondo Monetario Internacional y el Banco Mundial en el contexto del modelo económico globalizado y neoliberal, que una respuesta adecuada a las urgencias de la sociedad colombiana.

No obstante, incluso en un escenario fuertemente capitalista, la MTP está asociada, en los planes de desarrollo del gobierno actual, a una práctica educativa democrática, incompatible con lo que se ha denominado enseñanza tradicional o educación instruccional; además, se propondría para satisfacer las demandas de los sectores progresistas de la sociedad colombiana. Por lo tanto, la indicación de la práctica pedagógica que se deriva de la orientación de la MTP es que la escuela estará en condiciones de asegurar a los estudiantes las condiciones para llevar a cabo su aprendizaje de forma autónoma y no ser enseñados o colocados en el polo receptivo de una transmisión de conocimientos, concebida como 
contraria al aprendizaje. Se observa así la intención de conceptualizar la MTP articulada a las discusiones entre la educación y los procesos de globalización, haciendo referencia a los efectos en términos de la transformación de la educación.

Si bien, el análisis de los textos políticos y legislativos moldeó el abordaje del contexto de producción y, el contexto de influencia envolvió el estudio de la convergencia de propuestas y / o modelos internacionales, nacionales y locales y su articulación en la construcción de los textos que configuran la MTP, así como teorizaciones de analistas colombianos; el contexto de práctica representado en el estudio de caso se convirtió en el escenario para el análisis de cómo la política puede ser interpretada y adaptada para su materialización.

Fue posible entonces observar que hay, de hecho, una interrelación de cada contexto que alude al carácter integral y continuo del ciclo de políticas, conforme propuesto por Ball y sus colaboradores, configurándolos como contextos interrelacionados, que no tienen un orden temporal ni secuencial y, por lo tanto, no configuran etapas lineales.

En el proceso de construir problematizaciones en relación a las cuestiones propuestas por la investigación (políticas educativas como texto y como discurso), a partir de la lectura de las teorizaciones del filósofo Michel Foucault, apropiadas por Ball y sus coautores, sobre cuestiones de poder y discurso, nominamos como análisis del discurso el abordaje sistemático del material empírico: aunque no es propiamente una metodología, las proposiciones de Foucault y el diálogo con ellas establece que Ball y demás autores señalan principios que fueron fundamentales en tales análisis y justifican esta denominación. Fueron ellos, el reconocimiento que el discurso no solo describe, sino que también puede constituir realidades, así como la importancia de la atención a las cuestiones de poder que atraviesan los ejes de problematización definidos por la investigación.

Así, un primer argumento defendido viene de una visión de la escuela como un escenario que propicia, a partir de la propuesta de la MTP, por la formación de la persona autónoma responsable de su propio aprendizaje, que disputa con otra concepción de institución escolar, entendiéndola como un instrumento de concientización del alumno como ciudadano, en cuyo escenario el alumno no es formado exclusivamente para lograr el éxito personal, sino para llevar a cabo y liderar en la comunidad, las transformaciones que la sociedad necesita.

Con respecto a la acción transformadora atribuida a la escuela desde la MTP, la crítica que prevalece en el contexto empírico investigado propone la necesidad de pensar de manera diferente las formas en que se ha configurado el proceso escolar y, en particular, las formas de construcción del conocimiento, a partir de la MTP, para transformarlo en una fuerza social, aspecto que, según varios entrevistados durante la investigación, ha sido ignorado por la escuela, que se ve presionada a atender las demandas atribuidas por una visión marcadamente capitalista y neoliberal de la escuela y de la sociedad en general. También llamó la atención que la escuela, como se menciona en los testimonios de los participantes, es reconocida como un espacio importante para la socialización que, en base a experiencias 
como la MTP, privilegiaría el ejercicio de reflexión, análisis crítico, así como el debate / diálogo plural entre diferentes. Esto favorece que se respeten las diferencias y que sea posible encontrar innumerables oportunidades y vivencias. Es en esta dirección que se hace manifiesto el papel que aún se reconoce para la escuela en el proceso de socialización, un proceso que entendemos que debe fortalecerse desde caminos alternativos en una perspectiva que promueva por la reinvención de la escuela (Candau, 2015).

Con relación a los procesos más amplios que afectan la apropiación de MTP en el espaciotiempo investigado, otro punto a destacar se refiere a la discontinuidad de las políticas públicas educativas, aspecto que en este marco corresponde a la fragmentación de las acciones públicas, que incide sobre la educación escolar, afectando, en este caso, la efectividad de la MTP.

Podría pensarse que la marcada discontinuidad en la formulación de la política educativa en Colombia puede ser el resultado de diferentes perspectivas ideológicas, de los diferentes propósitos políticos, pero entendemos que también puede estar relacionado con una influencia muy fuerte en la forma en que se administra la educación, favoreciendo la centralización de las decisiones y las relaciones de poder. Del mismo modo, la falta de recursos materiales, que se presenta como un problema externo, también dificulta la viabilidad de la MTP, y entendemos que actualmente es parte de las opciones políticas impuestas en el país por las fuerzas políticas conservadoras.

En cuanto a las diversas formas en que se re-significa la MTP, en la institución en foco, se identificaron dos ejes principales: prácticas pedagógicas y conocimiento escolar. Con respecto a las prácticas pedagógicas, un aspecto que parece estar influenciado por la integración de la MTP se refiere a los efectos de las jerarquías escolares tradicionales, con énfasis importante en las formas en que los actores involucrados experimentan esta afectación, ya sea docente o alumno. También se debe tener en cuenta que, de acuerdo con los registros de observación, todavía hay resistencias más tradicionales de los profesores del colegio por la dificultad que afirman encontrar para conseguir relacionarse con el estudiante y con el conocimiento de esa manera.

Del mismo modo, las entrevistas han señalado los logros pedagógicos que se han logrado con la MTP, lo que indica que, con el tiempo y la experiencia, la estrategia también se está transformando en el escenario del Colegio. Esto es lo que se espera, en el marco teórico adaptado por esta investigación. En él, se entiende que las políticas se resignifican en el contexto de la práctica.

En el eje de enfoque denominado conocimiento escolar, nuestro objetivo fue cuestionar cómo se anuncian los elementos de innovación y superación de la perspectiva tradicional, haciéndose notoria una posición favorable en relación con el conocimiento que se fortalece mediante la integración de intereses, necesidades y realidades de los estudiantes. Se registró 
una referencia frecuente a un conocimiento escolar "descontextualizado", al tiempo que se enfatizó la innovación en relación con la enseñanza tradicional.

Aún con respecto a las ganancias pedagógicas que proporciona MTP en relación con el conocimiento escolar, es importante resaltar el valor que se atribuyó a la integración de las prácticas de investigación. Reconocer en este proceso que la voz de los jóvenes es una ganancia emocional y política que la MTP parece propiciar, especialmente si consideramos que la educación pública de Bogotá se enmarca en una amplia gama de diversidad y desigualdad social.

Concluimos que, a través del fortalecimiento de aspectos como la capacitación en investigación, así como la integración del contenido definido en la negociación con los estudiantes, en la dinámica del conocimiento escolar, la MTP puede aparecer como una estrategia que proporciona otras condiciones para favorecer la inserción profesional de los estudiantes en un contexto marcadamente neoliberal, al mismo tiempo que puede formar para la crítica social autónoma y creativa.

Finalmente, una conclusión digna de destacar de la MTP es la innegable posibilidad política y pedagógica de esta propuesta, aspecto que consideramos haber sido ampliamente destacado. Desde nuestro punto de vista, que se acerca más a las perspectivas críticas, la MTP tiene un gran potencial porque puede fortalecer los procesos educativos que se inscriben en la vida cotidiana de la escuela, articulando el conocimiento escolar con otros procesos sociales y favoreciendo la formación de estudiantes más autónomos y solidarios, capaces de construir historias y apostar por un mundo y una sociedad diferentes. Estudiantes cuyas voces se escuchan a través de procesos que se esfuerzan por ser participativos, personalizados y multidimensionales, y por tratar de hacer de la escuela un contexto más dinámico de interacción, de relaciones pedagógicas y de construcción de conocimiento, conforme fue ampliamente referido por estudiantes y maestros.

\section{CONSIDERACIONES FINALES}

Este artículo es el resultado de un análisis crítico que se realizó a partir de las discusiones, resultados y conclusiones obtenidas de la investigación reportada en él. En este análisis, destacar las contribuciones del abordaje del ciclo de políticas públicas propuesto por Stephen Ball y colaboradores que fundamento teóricamente una investigación de enfoque cualitativo, permitió reconocer que, desde la perspectiva de los enfoques cualitativos, el conocimiento es concebido como un proceso socialmente construido por los sujetos en sus interacciones cotidianas, mientras actúan sobre la realidad que configuran, transformándola y siendo transformados por ella también. Así, el mundo de los sujetos, los significados que atribuyen a sus experiencias diarias en su relación con la política en cuestión, su lenguaje, sus producciones culturales y sus formas de interacción constituyen ejes centrales de análisis que se abordaron en esta investigación. 
De manera que, la política recibida en la escuela (en sus representaciones multifacéticas) no llegaría transparente y unívoca en sus posibles significados, sino que, por el contrario, tendría una historia representada e interpretada. Además, sería una política que no caería en un vacío social o institucional si admitiéramos con Ball que tanto el texto de la política como sus lectores, así como el lugar donde se pone en práctica, le atribuyen significados en términos de sus historias, experiencias y lugares sociales. Tal comprensión en esta investigación implicó enfrentar el desafío de identificar las diversas posibilidades de significado de la MTP, a partir de los sentidos registrados en los textos normativos y documentos que subyacen a la propuesta, entendida como un "producto de múltiples influencias [que] involucran intenciones y negociaciones dentro del Estado y dentro del proceso de formación de las políticas" (Ball, 1994, p.13), así como los significados atribuidos por los actores participantes.

\section{Referencias}

André, M. (2013). O que é um estudo de caso qualitativo em educação? . Educação e Contemporaneidade, , 95 - 103.

Arfuch, L. (1995). La entrevista, una invensión dialógica. España: Ediciones Páidos Ibérica.

Arfuch, L. (2010). O espaço biográfico: dilemas da subjetividade contemporânea. Rio de Janeiro: Editora da UERJ.

Ball, S. (1994). Educational reform: a critical and post-structural approach. Buckingham: Open University Press.

Ball, S. J., Maguire, M., \& Braun, A. (2016). Como as escolas fazem as políticas, atação em escolas secundárias. Ponta Grosa - Paraná: UEPG.

Bowe, R., Ball, S., \& Gold, A. (1992). Reforming Education and Changing Schools: case studies in policy sociology. London: Routledge.

Candau, V. (2003). Pedagogias Críticas: ontem e hoje. Revista Novamerica, 58-61.

Dewey, J. (1979). Democracia e Educação: introdução à filosofia da educação (Quarta ed.). (R. Godofredo, \& A. Teixeira., Trads.) São Paulo: Editora Nacional.

Fairclough, N. (2001). Michael Foucault e a análise de discurso. Em N. Fairclough, Discurso e mudança social (pp. 61 - 81). Brasília: Editora Universidade de Brasília.

Fischer, R. (novembro/ 2001 de 2001). Foucault e a análise do discurso em educação. Cadernos de Pesquisa(114), 197-223.

Foucault. (1986). The archaeology of knowledge. New York: Tavistock Publications.

Knoll, M. (2012). I Had Made a Mistake: William H. Kilpatrick and the Projecto Method. Teachers Collage Record, 114(2). Acesso em 15 de 02 de 2017, disponível em http://www.tcrecord,org/content.asp?contenid16242 
Laville, C., \& Dionne, J. (1999). Manual de metodología da pesquisa em ciências humanas. . Porto Alegre: Artmed.

Lejeune, P. (1994). La autobiografía de los que no escriben. El pacto autobiográfico y otros estudios. Madrid: Megazul.

Mainardes, J., Santos, M., \& Tello, C. (2011). Análise de políticas: fundamentos e principais debates teórico-metodológicos. Em S. J. Ball, \& J. Mainardes, Políticas Educacionais: questões e dilemas. (pp. 143 - 172). São Paulo: : Cortez.

Maingueneau, D. (2015). Discurso e Análise do Discurso. São Paulo: Parábola.

Miñana, C. (Junio de 2008). "Tiempo para el aprendizaje": reformas educativas en Colombia y reconfiguración espacio-temporal de las escuelas. Revista Propuesta Educativa, FLACSO(29), $41-50$.

Rincón, Ó. (2010). Análisis de la política educativa actual en Colombia desde la perspectiva teórica de Pierre Bourdieu. Magistro. Revista de la Maestría en Educación de la Universidad Santo Tomás, 4(8). doi:10.15332/s2011-8643.2010.0008.02

Saraiva, K., \& Veiga-Neto, A. (2009). Modernidade Líquida, Capitalismo Cognitivo e Educação Contemporânea . Educação \& Realidade , 187-201.

Severino, A. (2007). Metodologia do Trabalho Científico. São Paulo: Cortez.

Stake, R. (2007). A arte de investigação com estudos de caso. Lisboa: Gulbenkian.

Tadeu Da Silva, T. (1997). El proyecto educacional moderno: ¿Identidad terminal? Em A. Veiga Neto, Crítica post-estructuralista y educación (pp. 273 - 290). Barcelona: Laertes. 